\title{
MISSILE INTERCEPTOR GUIDANCE AND CONTROL USING SECOND ORDER SLIDING MODES
}

\author{
${ }^{1}$ Yuri B. Shtessel, ${ }^{2}$ Ilya A. Shkolnikov and ${ }^{3}$ Arie Levant \\ ${ }^{I}$ Department of Electrical and Computer Engineering, The University of Alabama in \\ Huntsville, Huntsville, AL 35899 USA, phone: (256) 824-6164, fax: (256) 824-6803, \\ shtessel@ece.uah.edu \\ ${ }^{2} Z / I$ Imaging Corporation, Intergraph Company, 230 Business Park Blvd., Madison, AL \\ 35757, USA, ishkolnikov@ziimaging.com \\ ${ }^{3}$ Applied Mathematics Department, School of Mathematical Sciences, Tel-Aviv \\ University,Tel-Aviv69978, Israel, levant@post.tau.ac.il
}

\begin{abstract}
Abstract: The missile interceptors integrated guidance and control technology for achieving the hit-to-kill accuracy against targets performing evasive maneuvers including spiraling motion is developed on the basis of high (second) order sliding mode control (HOSM). The integration of guidance and flight control systems is achieved in a two-loop guidance and flight control system designed in the combined state space of engagement kinematics and vehicle dynamics. The designed guidance-control system performance is verified via computer simulations using miniature hypervelocity kinetic energy endo-atmospheric interceptor planar model. Copyright $@ 2005$ IFAC
\end{abstract}

Keywords: sliding mode control, missile guidance and control

\section{INTRODUCTION}

The miss distance as an ultimate performance criterion of a homing interceptor is crucially dependent on guidance, navigation and control (see Bezick, Rusnak, \& Gray, 1995; Garnell, \& East, 1977; and Zarchan, 1998) working together in a closed loop. This situation calls for the integrated design of all interceptor modules: sensor information processing and necessary data estimation, a homing guidance law, and flight control (autopilot). The primary goal of this work is to develop the enabling guidance and control technology for missile interceptors to achieve the hit-to-kill accuracy against targets performing evasive maneuvers including spiralling motion, using high order sliding mode control (HOSM) developed in works of Floquet, Barbot, \& Perruquetti (2003), Fridman (2001, 2003), Levant (1993,1998, 2001, 2003), and Orlov, Aguilar, \& Cadiou (2003).

The main concern of the paper is to use minimum possible information in order to achieve the goal (target intercept) in presence of uncertainties and disturbances acting in the homing loop. In this work, an integrated two-loop HOSM-based guidance and flight control system is designed to robustly enforce the target intercept in presence of target maneuvers, atmospheric disturbances, and dynamic uncertainty of airframe-actuator. The paper is organized as follows: Section 2 is dedicated to intercept strategy and a guidance law development based on a smooth second order sliding mode. In Section 3 integration of HOSM-based guidance and flight control system is presented. Simulations are discussed in Section 4. Conclusions are summarized in Section 5.

\section{INTERCEPT STRATEGY AND SLIDING MODE GUIDANCE}

\subsection{Introduction to Sliding Mode Guidance and Problem Formulation}

It's known (see Zarchan, 1998) that proportional navigation (PN) homing missile guidance can be derived as the optimal solution in mean-square sense, when there is no target maneuver, and only initial heading error should be nullified. In presence of a step-constant target maneuver augmented proportional navigation (APN) guidance is optimal for zero-lag guidance system. However, in reality the unmodeled series dynamics can cause instability of the homing loop that yields large miss distance. Optimal guidance law (OGL) effectively compensates for a single-lag frame dynamics in presence of a step-constant target maneuver modifying APN by using information of time-to-go and effective time-constant of the guidance system. However, OGL is difficult to derive and implement (see Zarchan, 1998).Recent application of sliding 
mode control (SMC) (see Utkin 1998) to the homing missile guidance (see Brierly and Longchamp, 1990; Babu, Sarma, and Swamy, 1994; Zhou, Mu, and Xu, 1999; Moon and Kim, 2000; Shkolnikov, Shtessel, et. al., 2000), resulted in a series of very effective algorithms in terms of smaller acceleration advantage required for intercept of weaving targets as compared to PN and APN guidance. Though SMC guidance doesn't use direct estimate of target acceleration, it's comparable to OGL. Since the guidance law must be smooth, all SMC guidance algorithms include smoothing procedures that yields partial loss of robustness to disturbances and uncertainties. In this work the problem is addresses in the frame of HOSM (see Levant 1993,1998, 2001, 2003; Shtessel, Shkolnikov, and Brown, 2003). A smooth asymptotic second order sliding mode control algorithm (SOSM) (see Shtessel, Shkolnikov, and Brown, 2003) is reassessed and is proved to be SOSM control. Next, the guidance algorithm based on a smooth SOSM control and SOSM controller/autopilot based on nonlinear dynamic sliding manifold (see Krupp, Shkolnikov, \& Shtessel, 2000) are used for designing an integrated guidance-flight-control system. The main idea developed in this work is to enforce the suitable closed-loop missile-target engagement kinematics using the input voltage signal to the actuator as control. In this case engagement kinematics and missile dynamics are integrated into one state space, and the intercept problem is transformed into the output regulation problem, which is addressed using SOSM-based control for the missile aerodynamic surface actuator.

\subsection{Intercept Strategy: Geometric Approach}

One can derive planar missile-target engagement kinematics without account for gravity as

$$
\left\{\begin{array}{l}
\dot{\mathbf{R}}=\mathbf{V}_{\mathbf{R}}, \quad \mathbf{V}_{\mathbf{R}}=\mathbf{V}_{\mathbf{T}}-\mathbf{V}_{\mathbf{M}}, \\
\dot{\mathbf{V}}_{\mathbf{R}}=\mathbf{A}_{\mathbf{R}}, \quad \mathbf{A}_{\mathbf{R}}=\mathbf{A}_{\mathbf{T}}-\mathbf{A}_{\mathbf{M}} .
\end{array}\right.
$$

such that $\mathbf{R}, \mathbf{V}_{\mathbf{R}}, \mathbf{A}_{\mathbf{R}}, \mathbf{V}_{\mathbf{T}}, \mathbf{V}_{\mathbf{M}}, \mathbf{A}_{\mathbf{T}}, \mathbf{A}_{\mathbf{M}} \in \mathfrak{R}^{2}$; $\mathbf{R}, \mathbf{V}_{\mathbf{R}}, \mathbf{A}_{\mathbf{R}}$ are range to the target and its first and second time-derivatives respectively; $\mathbf{V}_{\mathbf{T}}, \mathbf{A}_{\mathbf{T}}$ are target velocity and acceleration, $\mathbf{V}_{\mathbf{M}}, \mathbf{A}_{\mathbf{M}}$ are missile velocity and acceleration. The guidance command is missile normal acceleration, $n_{L}$. In polar coordinate system the missile-target relative position is presented by $\mathbf{R}=(r, \lambda)$, where $r=$ range along LineOf-Site (LOS), and $\lambda=$ LOS angle. The following state model (see Shkolnikov, Shtessel, et. al., 2000) of missile-target engagement process is obtained

$$
\left\{\begin{array}{l}
\dot{r}=V_{r}, \\
\dot{V}_{r}=r \omega_{\lambda}^{2}+A_{T r}-\sin \left(\lambda-\gamma_{M}\right) n_{L}, \\
\dot{\lambda}=\omega_{\lambda}, \\
\dot{\omega}_{\lambda}=\left(-2 V_{r} \omega_{\lambda}+A_{T \lambda}-\cos \left(\lambda-\gamma_{M}\right) n_{L}\right) / r,
\end{array}\right.
$$

where we consider $\omega_{\lambda}$ as a commanded output, missile normal acceleration as a control input, and projections of target acceleration along and orthogonal to $\operatorname{LOS}, A_{T r}, A_{T \lambda}$, are considered as unknown bounded disturbances. The system (2) can be written also as

$$
\left\{\begin{array}{l}
\dot{r}=V_{r}, \\
\dot{V}_{r}=V_{\lambda}^{2} / r+A_{T r}-\sin \left(\lambda-\gamma_{M}\right) n_{L}, \\
\dot{\lambda}=V_{\lambda} / r, \\
\dot{V}_{\lambda}=-V_{r} V_{\lambda} / r+A_{T \lambda}-\cos \left(\lambda-\gamma_{M}\right) n_{L},
\end{array}\right.
$$

where $V_{\lambda}=r \omega_{\lambda}$ is a transversal component of relative velocity in the reference frame rotating with LOS. It is well known that for a direct hit, it's necessary to keep $V_{r}<0$. It was shown in Shkolnikov, Shtessel, et. al., 2000 that direct hit can be achieved if $\omega_{\lambda}=0$ or $V_{\lambda}=0$. Another less aggressive hit-to-kill guidance strategy is known

$$
\omega_{\lambda}=c_{0} / \sqrt{r} \text { or } V_{\lambda}=c_{0} \sqrt{r} \text {, }
$$

where $c_{0}$ is some constant.

Now, the following guidance task can be formulated: Stabilize the system (2) or (3) on the manifold

$$
\sigma_{1}=\omega_{\lambda}=0, \text { or } \sigma_{1}=V_{\lambda}=0
$$

or

$$
\sigma_{2}=\omega_{\lambda}-c_{0} / \sqrt{r} .=0, \text { or } \sigma_{2}=V_{\lambda}-c_{0} \sqrt{r}=0
$$

by means of the normal acceleration (guidance) command $n_{L c}$. This command is usually followed by means of corresponding aerodynamic surface deflections that is treated as a control function compensating for the interceptor dynamics.

In the work of Shkolnikov, Shtessel, et. al., (2000) a different approach was proposed. After stabilization of (5) or (6) at zero the resulted compensated kinematics is derived. On the basis of this compensated kinematics a corresponding sliding surface is designed and stabilized by controlling the aerodynamic surface deflection. Doing this the guidance law in terms of $n_{L c}$ is dissolved in the sliding surface designed on the basis compensated kinematics of the interceptor. Unlike in the work Shkolnikov, Shtessel, et. al., (2000), where various continuous approximations of SMC were studied for the normal acceleration command, $n_{L c}$, design, in this work we propose to use HOSM-based control. The expected advantages are in increasing robustness and accuracy of hit-to-kill intercept.

\subsection{Prescribed Sliding Variable Dynamics}

Consider a SISO sliding variable dynamics given as

$$
\dot{\sigma}=f(x, t)+u,
$$

where $\sigma(x, t) \in \mathfrak{R}$ is the sliding quantity, such that $\sigma=0$ defines the system motion on the sliding surface, $u \in \mathfrak{R}^{1}$ is a control input that is supposed to be smooth, and $f(x, t)$ is an uncertain differentiable (smooth) nonlinear time-varying function, and $x \in \Re^{n}$ is a state vector of the system the sliding variable dynamics (7) is considered for. The drift term $f(x, t)$ is cancelled by means of a special observer to be developed further.

The following compensated $\sigma$-dynamics in (7) is considered 


$$
\left\{\begin{array}{l}
\dot{x}_{1}=-\alpha_{1}\left|x_{1}\right|^{(r-1) / r} \operatorname{sign}\left(x_{1}\right)+x_{2}, \\
\dot{x}_{2}=-\alpha_{2}\left|x_{1}\right|^{(r-2) / r} \operatorname{sign}\left(x_{1}\right),
\end{array}\right.
$$

where $x_{1}=\sigma$.

Definition. We call a system finite-time stable (Bacciotti, \& Rosier, 2001), if it is asymptotically stable with a finite settling time for any solution and initial conditions.

Lemma 1. Let $r \geq 2, \alpha_{1}, \alpha_{2}>0$. Then the system (8) is finite-time stable, and the settling time being a continuous and vanishing at the origin function of the initial conditions.

Proof. Consider the following Lyapunov function candidate:

$$
\begin{aligned}
& V=\frac{x_{2}^{2}}{2}+\int_{0}^{x_{1}} \alpha_{2}|z|^{(r-2) / r} \operatorname{sign}(z) d z= \\
& \frac{x_{2}^{2}}{2}+\frac{r}{2 r-2} \alpha_{2} x_{1}^{(2 r-2) / r}
\end{aligned}
$$

Its derivative is

$$
\begin{aligned}
& \dot{V}=\frac{\partial V}{\partial x} \cdot \dot{x}= \\
& {\left[\alpha_{2}\left|x_{1}\right|^{(r-2) / r} \operatorname{sign} x_{1}, x_{2}\right] \cdot\left[\begin{array}{l}
x_{2}-\alpha_{1}\left|x_{1}\right|^{(r-1) / r} \operatorname{sign} x_{1} \\
-\alpha_{2}\left|x_{1}\right|^{(r-2) / r} \operatorname{sign} x_{1}
\end{array}\right]=} \\
& \alpha_{2} x_{2} x_{1}^{(r-2) / r} \operatorname{sign}\left(x_{1}\right)-\alpha_{1} \alpha_{2}\left|x_{1}\right|^{(r-2) / r+(r-1) / r}- \\
& \alpha_{2} x_{2} x_{1}^{(r-2) / r} \operatorname{sign}\left(x_{1}\right)=-\alpha_{1} \alpha_{2}\left|x_{1}\right|^{(2 r-3) / r}
\end{aligned}
$$

Apply the La Salle theorem. The set $x:\{\dot{V}(x)=0\}$ consists of the axis $x_{1}=0$. It is easy to see that the only invariant set inside $x_{1}=0$ is the origin $x_{1}=x_{2}=0$. Thus, the asymptotic convergence of $x_{1}$ and $x_{2}$ to zero is assured. It is easy to see that system (8) is homogeneous with the dilation

$d_{\kappa}:\left(x_{1}, x_{2}\right) \mapsto\left(\kappa^{r} x_{1}, \quad \kappa^{r-1} x_{2}\right)$ and the negative homogeneity degree -1 . Following the results of Bacciotti, \& Rosier, (2001), the asymptotical stability implies here the finite-time stability and the continuity of the settling time function.

When the variables are sampled at discrete times $t_{i}$ with some sampling step $\tau>0$ the system (8) take on the form

$$
\begin{aligned}
& \dot{x}_{1}=-\alpha_{1}\left|x_{1}\left(t_{i}\right)\right|^{(r-1) / r} \operatorname{sign} x_{1}\left(t_{i}\right)+x_{2}\left(t_{i}\right) \\
& \dot{x}_{2}=-\alpha_{2}\left|x_{1}\left(t_{i}\right)\right|^{(r-2) / r} \operatorname{sign} x_{1}\left(t_{i}\right)
\end{aligned}
$$

where the current time satisfies the condition

$$
t_{i} \leq t<t_{i+1}=t_{i}+\tau
$$

Lemma 2. Let $r \geq 2$, and $\alpha_{1}, \alpha_{2}>0$. Then with $\tau>0$ all trajectories of the discrete-sampling system (9), (10) converge in finite time into some vicinity of the origin defined by the inequalities $\left|x_{1}\right|<\gamma_{1} \tau^{r},\left|x_{2}\right|<\gamma_{2}$ $\tau^{r-1}$ with $\gamma_{1}, \gamma_{2}>0$.

Proof. It is proven in Lemma 1 that with $\tau=0$ the system is finite-time stable. In particular all trajectories starting in some closed disk $D_{0}$ centred at the origin converge to the origin in some finite time
T. The right-hand sides of (8) are uniformly continuous in $D_{0}$, therefore, due to the continuous dependence on the right-hand side, with any $\tau$ smaller than some sufficiently small $\tau_{0}$ the trajectories of (9), (10) concentrate in some small closed disk $D_{1}{ }^{\prime} \subset D_{0}$. In their turn the trajectories of (8) starting from $D_{1}$ ' terminate at $O$ in time $T$. With sufficiently small $\tau_{1} \leq \tau_{0}, \tau \leq \tau_{1}$, the trajectories terminate in some other small disk $D_{1}{ }^{\prime}$, in time $T$, $D_{1}{ }^{\prime}, \subset D_{1}$ '. Let $D_{1}$ be some disk containing all the trajectories' segments starting from $D_{1}$ ' with $t$ varying in the range $[0, T], \tau \leq \tau_{1}, D_{1} \subset D_{0}$. Obviously, with $\tau \leq \tau_{1}$ any trajectory, which starts from $D_{0}$, enters $D_{1}$ in the time $T$ to stay there forever. Note that system (9), (10) is invariant with respect to the transformation

$G_{\kappa}:\left(t, \tau, x_{1}, x_{2}\right) \mapsto\left(\kappa t, \kappa \tau, \kappa^{r} x_{1}, \kappa^{r-1} x_{2}\right)$,

for any $\kappa>0$. There exists such $\kappa>1$ that $G_{\kappa} D_{1} \subset$ $D_{0}$. Thus, with $\tau \leq \kappa \tau_{1}$ all trajectories starting from $G_{\kappa} D_{0}$ enter $G_{\kappa} D_{1}$ in the time not longer than $\kappa T$ and then proceed into $D_{1}$ in time not longer than $T$ to stay there forever. In particular, it is true also for $\tau \leq \tau_{1}$. Thus a set sequence $D_{1} \subset D_{0} \subset G_{\mathrm{\kappa}} D_{0} \subset G_{\mathrm{\kappa}}^{2} D_{0} \subset \ldots$ covering the whole plane is constructed. Hence, with $\tau \leq \tau_{1}$ all trajectories enter $D_{1}$ in finite time to stay there forever.

Let $D_{1} \subset\left\{x_{1}, x_{2}|| x_{1}\left|\leq c_{1},\right| x_{2} \mid \leq c_{2}\right\}$, and let $\tau$ be some arbitrary sampling step. Applying $G_{\kappa}$ with $\kappa=$ $\tau / \tau_{2}$ achieve that with the sampling step $\tau$ any trajectory enters the region $\left\{x_{1}, x_{2}|| x_{1} \mid \leq\left(c_{1} / \tau_{2}{ }^{r}\right) \tau^{r}\right.$, ||$\left.x_{2} \mid \leq\left(c_{2} / \tau_{2}^{r-1}\right) \tau^{r-1}\right\}$ to stay there forever. Lemma 2 is proven.

Remark. Since $r>2$ can be any real number, Lemma 2 provides the first published example of a noninteger real-sliding order.

\subsection{Nonlinear disturbance observer}

The sliding variable dynamics (7) is sensitive to the unknown bounded term $f(\sigma(x(t), t), t)=g(t)$, which is assumed to be smooth with $|\dddot{g}| \leq L$. In order to estimate $g(t)$ the modified $2^{\text {nd }}$ order differentiator (Levant, 2003) is used

$$
\begin{aligned}
& \dot{z}_{0}=v_{0}, \quad v_{0}=-2 \cdot L^{1 / 3}\left|z_{0}-\sigma\right|^{2 / 3} \operatorname{sign}\left(z_{0}-f\right)+z_{1} \\
& \dot{z}_{1}=v_{1}, \quad v_{1}=-1.5 \cdot L^{1 / 2}\left|z_{1}-v_{0}\right|^{1 / 2} \operatorname{sign}\left(z_{1}-v_{0}\right)+z_{2}(12) \\
& \dot{z}_{2}=1.1 \cdot L \cdot \operatorname{sign}\left(z_{2}-v_{1}\right)
\end{aligned}
$$

and $g(t)=z_{1}(t)$ in a finite time, if the sliding variable $\sigma$ and control $u$ are measured without noise. Suppose that $\sigma$ and $u$ are measured with some Lebesgue-measurable noises bounded by $\varepsilon \geq 0$, then it is can be shown that there exists $\mu>0$ such that (Levant, 2003)

$$
\left|z_{1}(t)-g(t)\right| \leq \mu \varepsilon^{2 / 3}
$$

\subsection{Smooth High Order Sliding Mode Control}


Consider a sliding-variable dynamics (7) with smooth $f(\sigma, t)=g(t)$. The problem is to design control $u$ providing for the compensated $\sigma$-dynamics in (8). The result is given in the following Theorem.

Theorem. Let $r=3, \alpha_{1}, \alpha_{2}>0$, and $|\dddot{g}| \leq L$. Then the closed-loop system (7) controlled by the smooth control function

$u=z_{1}-\alpha_{1}\left|\sigma^{(r-1) / r} \operatorname{sign}(\sigma)-\alpha_{0} \int\right| \sigma^{(r-2) / r} \operatorname{sign}(\sigma) d \tau$

where $z_{1}$ is the output of the disturbance observer (11) is finite-time stable and

- If $\sigma(t)$ and $u(t)$ are measured with some Lebesgue-measurable noises bounded by $\varepsilon>0$, then inequality $|\sigma(t)| \leq \gamma \varepsilon$ is established in finite time for some constant $\gamma>0$ depending exclusively on the parameters of the controller.

- Let $\tau>0$ be the sampling interval and the noises be absent, then the inequality $|\sigma(t)| \leq \mu \tau^{3}$ is established in finite time for some constant $\mu>0$ depending exclusively on the parameters of the controller.

The proof follows from the overall homogeneity of the closed system (7), (12), (14) and is similar to the proof of Lemma 2. Let $w_{0}=z_{0}-\sigma, w_{1}=z_{1}-g, w_{1}=z_{2}-\dot{g}$. The following transformation is used:

$$
\begin{aligned}
G_{\kappa}: & \left(t, \tau, \varepsilon, x_{1}, x_{2} w_{0}, w_{1}, w_{2}\right) \\
& \mapsto\left(\kappa t, \kappa \tau, \kappa^{3} \varepsilon, \kappa^{3} x_{1}, \kappa^{2} x_{2}, \kappa^{3} w_{0}, \kappa^{2} w_{1}, \kappa w_{2}\right) .
\end{aligned}
$$

\subsection{Smooth Second Order Sliding Mode Guidance}

At this moment, the chosen intercept strategy transforms the intercept problem to an output regulation problem, which can be considered as a fundamental problem in geometric methods of control synthesis. The control goal of the output regulation problem is to stabilize $\sigma_{i}, i=1,2$ and to satisfy the condition $\forall t \geq t_{o}, V_{r}<0$ provided given limits on control input, $\left|n_{L}\right| \leq n_{L \text { max }}$, and known bounds of uncertainties variations. We wish to solve this problem without explicit estimation of uncertainties but to apply robust control methods, where control action will automatically counteract the influence, which steers the system away from specified path. A number of solutions to this problem using SMC theory is developed in the last decade. Each of them can be used to create a reference closed-loop model to be enforced in presence of the unmodeled at this stage vehicle dynamics of a homing interceptor. The important issue here is to design a continuous/smooth control, $n_{L c}(t)$, to be enforced for the actual missile normal acceleration $n_{L}(t)$. That's why absolute robustness to uncertainties and finite time convergence are traded for approximation of an ideal discontinuous SMC law by a linear saturation function (see Moon, \& Kim, 2000; Shkolnikov, Shtessel, et. al., 2000). In this case the output is robustly regulated to a small domain of convergence around zero.
In this paper, we employ smooth second order sliding mode control (14) with $r=3$. From (6) the $\sigma$ dynamics is identified as (we omit the subscript)

$\dot{\sigma}=-V_{r} V_{\lambda} / r+A_{T, \lambda}-c_{0} V_{r} /(2 \sqrt{r})-\cos \left(\lambda-\gamma_{M}\right) n_{L}$

so the commanded acceleration, $n_{L c}$, for the

interceptor normal acceleration $n_{L}$ is selected to be

$$
\begin{aligned}
& n_{L c}=\frac{1}{\cos \left(\lambda-\gamma_{M}\right)}\left(\alpha_{1} \mid \sigma^{2 / 3} \operatorname{sign} \sigma+\right. \\
& \alpha_{0} \int \mid \sigma^{1 / 3} \operatorname{sigh}(\sigma) d \tau-\frac{V_{r} V_{\lambda}}{r}-\frac{c_{0} V_{r}}{2 \sqrt{r}}+\hat{A}_{\text {smooth } T, \lambda},
\end{aligned}
$$

where $\hat{A}_{\text {smooth }, \lambda}$ is estimated using (12).

The homing (when a seeker is activated and the designed guidance law is executed) close loop interceptor kinematics (3), (6), (14) is derived as follows:

$$
\left\{\begin{array}{l}
\dot{r}=V_{r}, \\
\dot{V}_{r}=c_{0}^{2}+A_{T r}-\sin \left(\lambda-\gamma_{M}\right) n_{L}, \\
\dot{\lambda}=c_{0} / \sqrt{r}, \\
V_{\lambda}=c_{0} \sqrt{r},
\end{array}\right.
$$

Since the initial condition $V_{r}(0)=M<<0$, it is easy to identify $c_{0}$ such that $V_{r}(t)<0 \forall t \leq t_{g o}$, where $t_{g o}$ is time required for the interceptor to hit the target. So, the guidance law (15) based on asymptotic smooth second order sliding mode control inevitably guarantees hit-to-kill intercept.

\section{INTEGRATION OF GUIDANCE AND FLIGHT CONTROL SYSTEMS: 2-LOOP SECOND ORDER SMC APPROACH}

\subsection{Missile Interceptor Dynamics}

The following pitch plane model of kinetic energy kill vehicle (KEKV) without account for gravity (see Shkolnikov, Shtessel, et. al., 2000) is considered assuming the model to be affine in controls, i.e. in case of only aerodynamic control ( $\delta_{e}$ is a virtual deflection, which is allocated by the fin's mixing logic) we have

$$
\begin{aligned}
& {\left[\begin{array}{l}
\dot{\alpha} \\
\dot{V} \\
\dot{\theta} \\
\dot{q}
\end{array}\right]=\left[\begin{array}{l}
-\frac{X}{m V} \sin \alpha+\frac{Z}{m V} \cos \alpha+q \\
\frac{X}{m} \cos \alpha+\frac{Z}{m} \sin \alpha \\
q \\
\frac{\bar{q} S l}{I_{y y}}\left(C_{m_{o}}(\cdot)+\Delta C_{m}(t)\right)
\end{array}\right]+\left[\begin{array}{c}
0 \\
0 \\
0 \\
\frac{\bar{q} S l}{I_{y y}} C_{m}^{\delta_{e}}(\cdot)
\end{array}\right] \cdot \delta_{e}} \\
& n_{L}=\frac{\bar{q} S}{m} C_{L}(\alpha, \ldots)=-A_{x} \sin \alpha+A_{z} \cos \alpha=\dot{\gamma}_{M} \cdot V
\end{aligned}
$$

where $\gamma_{M}=\theta-\alpha$ is the flight path angle, $n_{L}$ is a normal acceleration, $A_{x}=\frac{X}{m}, A_{z}=\frac{Z}{m}, \quad \bar{q}=\frac{1}{2} \rho V^{2}$, $X=\bar{q} S\left(C_{x}(\cdot)+\Delta C_{x}(t)\right), \quad Z=\bar{q} S\left(C_{z}(\cdot)+\Delta C_{z}(t)\right)$, $C_{m}\left(\alpha, \dot{\alpha}\right.$, Mach $\left., q, \delta_{e}, \ldots\right)=C_{m_{o}}(\cdot)+C_{m}^{\delta_{e}}(\cdot) \cdot \delta_{e}, \quad$ and $\Delta C_{x}(t), \Delta C_{z}(t), \Delta C_{m}(t)$ are the external disturbances. It's also assumed that the model (18) is of minimum phase. In case of aerodynamic control, it means that $\frac{\partial C_{L}}{\partial \delta_{e}}=0, \frac{\partial C_{D}}{\partial \delta_{e}}=0$, which is true, if the extra small 
fins are employed. The actuators have the highly nonlinear dynamics with the relative degree equal to one, which, after mixing control commands to individual actuators actuator, can be presented as

$$
\dot{\delta}_{e}=-a\left(\delta_{e}, t\right)\left(\delta_{e}-u\right),
$$

where the nonlinear uncertain term $a\left(\delta_{e}, t\right)$ is allowed to be non-smooth but bounded.

\subsection{Model Behavior of the Homing Loop}

Now, the problem is to design the control input to the actuator, $u(t)$, in order to enforce the given closed loop performance of a homing loop robustly to uncertainty of vehicle dynamics. Under the second order smooth asymptotic SMC guidance (15), (16) the closed-loop $V_{\lambda}$-dynamics are

$$
\dot{V}_{\lambda}=c_{0} V_{r} /(2 \sqrt{r})
$$

It was shown in the work of Shkolnikov, Shtessel, et. al., 2000) by simulations that even in presence of uncompensated $A_{T, \lambda}$ the closed-loop $V_{\lambda}$-dynamics enforced by the traditional SMC guidance can provide for the reliable target intercept. The model behavior (21) should be robustly enforced by control $\mathrm{u}$ in presence of vehicle dynamics uncertainties and disturbances and target maneuvers represented by target acceleration components $A_{T, \lambda}$ and $A_{T, r}$. This problem is addressed via SOSM approach (see Levant 1993, 1998, 2001, 2003; Fridman 2001, 2003) and a two-loop controller structure (see Shtessel, Buffington, and Banda, 1999).

\subsection{Outer Loop SMC Design}

Now we consider the composite state space of the systems (3) and (18). If we hold

$$
\sigma_{0}=\dot{V}_{\lambda}-c_{0} V /(2 \sqrt{r})
$$

at zero, then even under small residual perturbation, $\left|\sigma_{0}(t)\right| \leq N$, an acceptable closed-loop performance that leads to the hit-to-kill intercept can be achieved (see Shkolnikov, Shtessel, et. al., 2000).

Following the SMC approach, we call the constraint (22) to be kept at zero as the sliding manifold in the outer loop, where $\sigma_{0}$ is the sliding quantity. To stabilize $\sigma_{0}$ to zero, its dynamics is identified

$$
\begin{gathered}
\dot{\sigma}_{0}=-\frac{\left(\dot{V}_{r} V_{\lambda}+V_{r} \dot{V}_{\lambda}\right) r-V_{r}^{2} V_{\lambda}}{r^{2}}+\dot{A}_{T, \lambda}- \\
\sin \left(\lambda-\gamma_{M}\right)\left(\frac{V_{\lambda}}{r}-\frac{n_{L}}{V}\right) n_{L}-\cos \left(\lambda-\gamma_{M}\right) \dot{n}_{L}
\end{gathered},
$$

One can rearrange the terms in (23) and write it in the short cut notation as

$$
\dot{\sigma}_{0}=\varphi-\cos \left(\lambda-\gamma_{M}\right) \dot{n}_{L}
$$

where

$$
\begin{aligned}
& \varphi=\varphi\left(\dot{V}_{r}, V_{r}, \dot{V}_{\lambda}, V_{\lambda}, r, V, \dot{A}_{T, \lambda}, \lambda, \gamma_{M}, n_{L}\right)= \\
& -\frac{\left(\dot{V}_{r} V_{\lambda}+V_{r} \dot{V}_{\lambda}\right) r-V_{r}^{2} V_{\lambda}}{r^{2}}+\dot{A}_{T, \lambda}-\sin \left(\lambda-\gamma_{M}\right)\left(\frac{V_{\lambda}}{r}-\frac{n_{L}}{V}\right) n_{L}
\end{aligned}
$$

Designing a smooth second order SMC (13) in terms of $\dot{n}_{L c}$ ("jerk" command), we obtain the following command on missile normal jerk $\dot{n}_{L c}$

$\dot{n}_{L c}=\frac{1}{\cos \left(\ell-\gamma_{M}\right)}\left(\bar{\alpha}_{1}\left|\sigma_{0}\right|^{2 / 3} \operatorname{sig} \sigma_{0}+\left.\bar{\alpha}_{0} \int \sigma_{0}\right|^{1 / 3} \operatorname{sig}\left(\left(\sigma_{0}\right) d \tau\right)\right.$,
Although, the inner loop second order sliding mode control can be designed to track $\dot{n}_{L}$, it is more convenient to track $q_{c}$, a command for the pitch rate, since the pitch rate is measuring accessible. From the interceptor dynamics (18) we identify

$$
q=\frac{1}{V}\left(T_{\alpha} \dot{n}_{L}+\int \dot{n}_{L} d \tau\right)
$$

where $V$ is an interceptor speed (magnitude), and $T_{\alpha}$ is known as the turning rate coefficient

$$
T_{\alpha}=\frac{\alpha}{\dot{\gamma}_{M}}=V\left(\frac{m}{\bar{q} S}\right)\left(\frac{\partial C_{L}}{\partial \alpha}(\cdot)\right)^{-1}
$$

Considering $T_{\alpha}$ as a known slowly varying quantity (that is true for the majority of intercept scenarios), we finally obtain the following profile for the interceptor pitch rate to follow

$$
\begin{aligned}
& q_{c}(t)=\frac{1}{V \cos \left(\lambda-\gamma_{M}\right)}\left[T _ { \alpha } \left(\bar{\alpha}_{1}\left|\sigma_{0}\right|^{2 / 3} \operatorname{sign}\left(\sigma_{0}\right)+\right.\right. \\
& \left.\bar{\alpha}_{0} \int\left|\sigma_{0}\right|^{1 / 3} \operatorname{sign}\left(\sigma_{0}\right) d \tau+\hat{\varphi}_{\text {smooth }}\right) d \tau+ \\
& \left.\int\left(\bar{\alpha}_{1}\left|\sigma_{0}\right|^{2 / 3} \operatorname{sign} \sigma_{0}+\bar{\alpha}_{0} \int\left|\sigma_{0}\right|^{1 / 3} \operatorname{sign}\left(\sigma_{0}\right) d \tau\right) d \tau\right]
\end{aligned}
$$

Implementing the pitch rate command it necessary to compute $\dot{V}_{\lambda}$, since $\sigma_{0}=\dot{V}_{\lambda}-c_{0} V_{r} /(2 \sqrt{r})$. It can be done using the first order exact differentiator (see Levant 1998, 2003), or the first order robust-to noise differentiator based on nonlinear dynamic sliding manifold (NDSM) (see Krupp, Shkolnikov, \& Shtessel, 2000), where NDSM is developed to incorporate a low-pass nonlinear filter in the observer structure producing a robust to noise differentiator. A robust differentiator of a given signal, $x(t)$, polluted with noise, has the form

$$
\begin{cases}\ddot{\hat{x}}=\rho_{o} \operatorname{sign}\left(J_{o}\right), & J_{o}=\chi+e, \\ \dot{\chi}=b \frac{e}{|e|^{0.5}}-a \frac{(\chi+e)}{|\chi+e|^{0.5}}, & e=x(t)-\hat{x},\end{cases}
$$

where the parameters should be appropriately selected in accordance with the upper boundary for $|\ddot{x}(t)| \leq L$. Apparently, when the 2-sliding mode, $J_{o}=\dot{J}_{o}=0$, is established, we have the estimation error dynamics $\dot{e}=-b|e|^{0.5} \operatorname{sign}(e)$, which converges to zero in a finite time. Thus, a command on missile maneuver is obtained in terms of pitch rate command $q_{c}$. To obtain this command we had to consider the composite state space of engagement kinematics and vehicle dynamics. The inner loop control is designed next to robustly enforce $q_{c}$ in presence of uncertainties and disturbances.

\subsection{Inner Loop Second Order SMC Design}

The regulated output in the inner loop is the pitch tracking error

$$
e_{q}=q_{c}(t)-q \text {. }
$$

From (18) and (20) we determine that the relative degree of the input-output dynamics for $e_{q}$

$$
\ddot{e}_{q}=\varphi_{1}(\cdot)-\frac{T_{\alpha}}{V}\left(T_{\alpha}\right)^{-1} \omega_{n}^{2}(\cdot) a(.) u,
$$


is equal to two, where $\varphi_{1}($.$) is a nonlinear, time-$ varying uncertain bounded term. Thus, one can apply the SOSM approach (see Levant 1993, 1998, 2003) to provide for finite time convergence of the tracking error $e_{q}$ to zero robustly to time-varying additive uncertainty $\varphi_{1}(\cdot)$ and multiplicative uncertainties of airframe dynamics, $\omega_{n}^{2}=\omega_{n}^{2}(\cdot)=\frac{\bar{q} S l}{I_{y y}} C_{m}^{\delta_{e}}(\cdot) \quad\left(\omega_{n}\right.$ is an equivalent undamped natural frequency of airframe) and actuator dynamics $a=a\left(\delta_{e}, t\right)$ (actuator bandwidth). The only requirement is to know the limit of $\varphi_{1}(\cdot)$ variations, and the nominal value and sign of the term $\frac{1}{V} \omega_{n}^{2}(\cdot) a(\cdot)$. For instance, SOSM that provides convergence of $e_{q}$ to zero in finite time in presence of bounded uncertainties can be designed in a format (see Levant 1993, 2001)

$$
u=\frac{V}{\omega_{n}^{2}} \alpha \operatorname{sign}\left(\dot{e}_{q}+\left|e_{q}\right|^{1 / 2} \operatorname{sign}\left(e_{q}\right)\right)
$$

In order to avoid differentiation of $e_{q}$ control (31) can be also designed based on NDSM (see Krupp, Shkolnikov, \& Shtessel, 2000) as follows:

$$
\begin{aligned}
& u=\frac{V}{\omega_{n}^{2} a} \rho \operatorname{sign}(J), J=e_{q}+\chi \\
& \dot{\chi}=\beta_{1}\left|e_{q}\right|^{0.5} \operatorname{sign}\left(e_{q}\right)-\beta_{2}|J|^{0.5} \operatorname{sign}(J)
\end{aligned}
$$

where $J$ is the nonlinear dynamic sliding quantity, the nominal values for uncertainties $\omega_{n}^{2}(\cdot)$ and $a(\cdot)$ are used, and the coefficient $\rho$ is selected according to the upper absolute limit for additive uncertainty $\varphi_{1}(\cdot)$. Thus, using only output feedback $e_{q}$, the control voltage (32) to the actuator (20) provides for the output $e_{q}$ convergence to zero in a finite time.

\section{SIMULATION EXAMPLE}

Simulation results of KEKV using smooth SOSM guidance and SOSM/NDSM-based autopilot are available and are omitted for brevity.

\section{CONCLUSIONS}

An integrated two-loop guidance and flight control system is designed based on second order sliding mode control to robustly enforce hit-to-kill guidance strategy in presence of target maneuvers, atmospheric disturbances, and dynamic uncertainty of airframe-actuator. The zero miss target intercept is achieved with the reduced acceleration ratio requirements due to prolonged stability of the homing loop.

\section{REFERENCES}

Bacciotti A. \& Rosier L., (2001). Liapunov Functions and Stability in Control Theory, Lecture notes in control and information sciences, 267, Springer-Verlag, New-York.

Babu, K., Sarma, I., and Swamy, K., (1994). Switched Bias Proportional Navigation for Homing Guidance Against Highly Maneuvering Target. Journal of Guidance, Control and Dynamics, 17(6), 1357-1363.

Brierly, S.D., \& R. Longchamp, (1990). Application of Sliding Mode Control to Air-Air Interception Problem.
IEEE Transaction on Aerospace and Electronic Systems, No.2, 306-325.

Floquet, T., Barbot, J.-P., \& Perruquetti, W. (2003). Higher-order sliding mode stabilization for a class of nonholonomic perturbed systems. Automatica, 39, 1077 1083.

Fridman, L., (2001). An averaging approach to chattering. IEEE Transactions of Automatic Control, 46, 1260 1265.

Fridman, L. (2003). Chattering analysis in sliding mode systems with inertial sensors, International Journal of Control, 76 (9/10), 906-912.

Garnell, P., and East, D. J. (1977). Guided Weapon Control Systems, Oxford: Pergamon Press.

Krupp D. R., Shkolnikov I. A., and Shtessel Y. B., (2000). 2-Sliding Mode Control for Nonlinear Plants with Parametric and Dynamic Uncertainties. Proceedings of AIAA Guidance, Navigation and Control Conference, Denver, CO, Paper AIAA-2000-3968.

Levant A. ( Levantovsky L.V.) (1993). Sliding order and sliding accuracy in sliding mode control. International Journal of Control, Vol. 58, No. 6, 1247-1263,

Levant, A. (1998). Robust exact differentiation via sliding mode technique. Automatica, (34), 3, 379-384.

Levant A., (2001). Universal SISO sliding-mode controllers with finite-time convergence. IEEE Transactions on Automatic Control, 46(9), 1447-1451.

Levant A., (2003). Higher-order sliding modes, differentiation and output-feedback control. International Journal of Control, 76 (9/10).

Moon, J., Kim, Y., (2000). Design of missile guidance law via variable structure control. Proceedings of AIAA Guidance, Navigation and Control Conference, Denver, CO, Paper AIAA-2000-4068.

Orlov, Y., Aguilar, L., and Cadiou, J. C. (2003). Switched chattering control vs. backlash/friction phenomena in electrical servo-motors. International Journal of Control, $76(9 / 10), 959$ - 967.

Shkolnikov, I. A., Shtessel, Y. B., Lianos, D. P., and Thies, A. T., (2000), Robust Missile Autopilot Design via HighOrder Sliding Mode Control. Proceedings of AIAA Guidance, Navigation, and Control Conference, AIAA Paper 2000-3968.

Y. Shtessel, I. Shkolnikov and M. Brown, (2003). An Asymptotic Second-Order Smooth Sliding Mode Control," Asian Journal of Control, 4, No. 5, 498-504.

Shtessel, Y., Buffington, J., and Banda, S., (1999). Multiple Time Scale Flight Control Using Reconfigurable Sliding Modes, AIAA Journal on Guidance, Control, and Dynamics, (22), 6, 873-883.

Utkin, V., Guldner, J., and Shi, J. (1999). Sliding Modes in Electromechanical Systems, Taylor and Francis, London. Zarchan P., (1998). Tactical and Strategic Missile Guidance. Vol. 176, Progress in Astronautics and Aeronautics, AIAA Publications.

Zhou, D., Mu, C. \& Xu, W. (1999). Adaptive SlidingMode Guidance of a Homing Missile. Journal of Guidance, Control and Dynamics, (22), 4, 589-594. 